\title{
Proteins in Mixed Solvents: A Molecular-level Perspective
}

\author{
Brian M. Baynes, Daniel I. C. Wang, and Bernhardt L. Trout \\ Department of Chemical Engineering, M.I.T., 77 Massachusetts Ave., Cambridge, MA 02139, USA
}

\begin{abstract}
We present a statistical mechanical approach for quantifying thermodynamic properties of proteins in mixed solvents. This approach, based on molecular dynamics simulations which incorporate all atom models and the theory of preferential binding, allows us to compute transfer free energies with experimental accuracy and does not incorporate any adjustable parameters. Specifically, we applied our approach to the model proteins RNase A and T1, and the solvent components water, glycerol, and urea. We found that the observed differences in the binding of glycerol and urea to RNase $\mathrm{T} 1$ and $\mathrm{A}$ are predominantly a consequence of density differences in the first coordination shell of the protein with the cosolvents, but the second solvation shell also contributes to the overall binding coefficients. The success of this approach in modeling preferential binding indicates that it incorporates the important underlying physics of proteins in mixed solvent systems and that the difficulty in quantitative prediction to date can be surmounted by explicitly incorporating the complex protein-solvent and solvent-solvent interactions.
\end{abstract}

Index Terms-Glycerol, molecular dynamics, preferential binding, ribonuclease, urea

\section{INTRODUCTION}

Proteins are seldom solvated by pure water. Other solvent components, such as buffer salts and stabilizers, are ubiquitous in the laboratory and in formulations of therapeutic proteins. Similarly, intracellular solutions are crowded with many types of proteins, metabolites, nucleic acids, osmolytes, and other molecules. The presence of these other components, hereafter called "cosolvents," generally alters protein equilibria and reaction kinetics by perturbing the chemical potential of the protein system. Cosolvents perturb the chemical potential of the protein system by associating either more strongly or more weakly with the protein than water. This phenomenon, called "preferential binding" [1], is of great interest because it governs the physical and chemical properties of proteins.

When a cosolvent $(\mathrm{X})$ is added to an aqueous protein solution, it alters the chemical potential of the protein $\left(\mu_{P}\right)$ via the following relationship [2]:

$$
\begin{aligned}
\Delta \mu_{P}^{t r} & =\int_{0}^{m_{X}}\left(\frac{\partial \mu_{P}}{\partial m_{X}}\right)_{m_{P}} d m_{X} \\
& =-\int_{0}^{m_{X}}\left(\frac{\partial \mu_{X}}{\partial m_{X}}\right)_{m_{P}}\left(\frac{\partial m_{X}}{\partial m_{P}}\right)_{\mu_{X}} d m_{X}
\end{aligned}
$$

B. M. Baynes, D. I. C. Wang, and B. L. Trout can be reached by electronic mail at baynes@mit.edu, dicwang@mit.edu, and trout@mit.edu, respectively.

This work was supported by the United States National Institutes of Health Biotechnology Training Program and the National University of Singapore. where $\Delta \mu_{P}^{t r}$ is the transfer free energy of the protein from pure water into the mixed solvent system, $m$ is molality, and subscripts $X$ and $P$ identify the cosolvent and protein respectively. Two partial derivatives appear in equation 2 . The first captures the dependence of the cosolvent chemical potential on cosolvent molality and can be evaluated by experiments on a binary mixture of cosolvent and water $\left(m_{P} \rightarrow 0\right)$. The second partial derivative is the "preferential binding coefficient," $\Gamma_{X P}$ :

$$
\Gamma_{X P} \equiv\left(\frac{\partial m_{X}}{\partial m_{P}}\right)_{\mu_{X}}
$$

The preferential binding coefficient is a way in which binding can be defined thermodynamically. It is also particularly useful when binding is weak. The preferential binding coefficient is a measure of the excess number of cosolvent molecules in the domain of the protein per protein molecule (Figure 1). The connection between the thermodynamic definition (equation 3) and the intuitive notion of binding (local excess number of molecules) comes from statistical mechanics, where it can be shown that [3], [4]:

$$
\Gamma_{X P}=\left\langle n_{X}^{I I}-n_{W}^{I I}\left(\frac{n_{X}^{I}}{n_{W}^{I}}\right)\right\rangle
$$

In the above equation, $n$ denotes the number of a specific type of molecule (subscript $X$ for the cosolvent and subscript $W$ for water) in a certain domain (superscript $I$ for a bulk volume outside of the vicinity of the protein and superscript $I I$ for a volume in the protein vicinity), and angle brackets denote an ensemble average. Note that $\Gamma_{X P}$ is independent of the choice of the boundary between the domains, as long as the boundary is far enough from the protein.

If the cosolvent concentration is higher in the vicinity of the protein than in the bulk, $\Gamma_{X P}$ is greater than zero, and $\mu_{P}$ is lower in the presence of the cosolvent than in its absence. Denaturants such as urea and guanidinium chloride exhibit this type of binding behavior. The reverse is true for sugars, such as trehalose. In trehalose solutions, there is generally a deficiency of trehalose and an excess of water in the vicinity of the protein. For this "preferential hydration" case, $\Gamma_{X P}$ is less than zero, and $\mu_{P}$ is higher in the presence of the cosolvent.

Thirty years ago, Timasheff pioneered the use of highprecision densitometry to measure preferential binding coefficients for protein-cosolvent systems [2], [5], [6], [7]. More recently, differential scanning calorimetry (DSC) [8] and vapor pressure osmometry (VPO) [9] have been used to the same end. Preferential binding coefficients are rigorous thermodynamic quantities and are related to virial coefficients, activity 


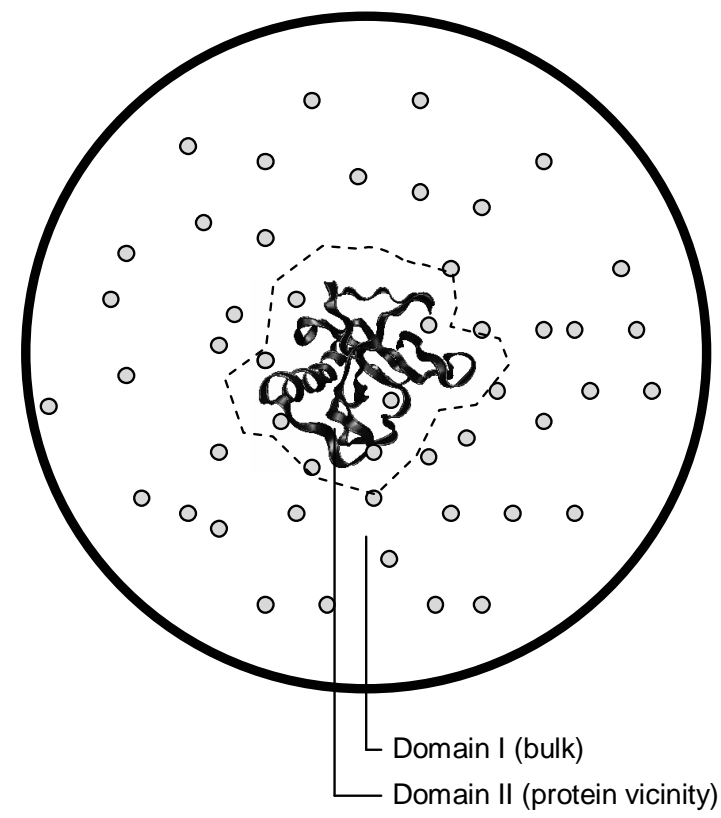

Fig. 1. Physical interpretation of the preferential binding coefficient Interactions of solvent molecules with the protein at the protein-solvent interface generally induce solvent concentration differences in the local (II) and bulk (I) domains. $\Gamma_{X P}$ is the thermodynamic measure of the number of cosolvent molecules bound to the protein, or in other words, the excess number of cosolvent molecules in the vicinity of the protein versus the number of cosolvent molecules in an equivalent volume of bulk solution.

coefficients, and free energies via standard thermodynamic relations for multi-component solutions [10].

Experimental studies by the above methods have led to some generalizations about preferential binding coefficients:

1) $\Gamma_{X P}$ may be positive or negative, indicating that interactions of the protein and cosolvent are favorable or unfavorable, respectively.

2) $\Gamma_{X P}$ is proportional to cosolvent molality at low concentration of cosolvent (often as high as $m_{X} \sim 1 \mathrm{~m}$ and higher) [9], [11], [12].

3) $\Gamma_{X P}$ is roughly proportional to the protein-solvent interfacial area [2].

The second generalization above, together with the fact that many binary mixtures of cosolvent and water $\left(m_{P} \rightarrow 0\right)$ are nearly ideal at low concentration of cosolvent, leads to a useful simplification of equation 2 :

$$
\begin{aligned}
\Delta \mu_{P}^{t r} & =-\int_{0}^{m_{X}}\left(\frac{\partial R T \ln m_{X}}{\partial m_{X}}\right)\left(\frac{\Gamma_{X P}}{m_{X}}\right) m_{X} d m_{X}(5) \\
& =-R T\left(\frac{\Gamma_{X P}}{m_{X}}\right) \int_{0}^{m_{X}} d m_{X} \\
& =-R T \Gamma_{X P}
\end{aligned}
$$

Equation 7 provides a simple and convenient link between preferential binding coefficients and free energies. This relation leads to the useful rule that when $\Gamma_{X P}$ is proportional to $m_{X}$, for each cosolvent molecule that preferentially interacts with the protein, the protein's free energy is reduced by approximately $0.6 \mathrm{kcal} / \mathrm{mol}$ at $25^{\circ} \mathrm{C}$. The simplicity of this relation is a natural result of the close relationship between $\Gamma_{X P}$ and a second virial coefficient.

To be able to predict preferential binding coefficients and understand their origins, the above thermodynamic framework and general observations must be augmented by a mechanistic model. Several such models have been presented in the literature, including models based on the binding polynomial or statistical mechanical partition function, solvent-cosolvent exchange at defined sites, cosolvent partitioning between the local and bulk domains, group contribution methods for estimating transfer free energies.

The most general model of cosolvent binding hitherto presented comes from considering an equilibrium of all possible protein-cosolvent complexes, from which it can be shown that [13]:

$$
\Delta \mu_{P}^{t r}=-R T \ln \left(1+\sum_{i} \sum_{j} K_{i j} m_{W}^{i} m_{X}^{j}\right)
$$

where $K_{i j}$ is the equilibrium constant for a reaction of a protein molecule, $i$ molecules of water, and $j$ molecules of cosolvent into a complex. While this model is completely general, its utility is limited because it is not possible to determine experimentally the many $K_{i j}$ parameters present in equation 8.

Schellman's site exchange model [4] provides a way to simplify this general expression to a form containing a single parameter. This model treats binding as a family of proteinsolvent exchange reactions such as:

$$
P \cdot W_{i}+X \rightarrow P \cdot X+i W
$$

where $P$ is the protein, $W$ is water, $X$ is cosolvent, and $i$ is the exchange stoichiometry. The simplification requires the assumptions that $1: 1$ exchange reactions $(i=1)$ occur on a fixed number of identical, independent sites and that the sites are far from saturation with cosolvent (i.e. the apparent dissociation equilibrium constant for each site is well above the cosolvent concentration). The number of sites, $n$, is approximated by the number of water molecules present in a monolayer around the protein. These simplifications reduce equation 8 to:

$$
\Delta \mu_{P}^{t r}=-n R T\langle K\rangle m_{X}
$$

where $\langle K\rangle$ is the average equilibrium constant of binding at a single site. The single parameter $\langle K\rangle$ can then be determined from an experimental measurement of $\Gamma_{X P}$. When equation 7 holds, the relation between $\langle K\rangle$ and $\Gamma_{X P}$ is simply:

$$
\langle K\rangle=\Gamma_{X P} / n m_{X}
$$

Values of $\langle K\rangle$ for different proteins in this linear regime are roughly equal [14]. $\langle K\rangle$ cannot, however, be determined without knowledge of $\Gamma_{X P}$ or other free energy data on the particular cosolvent system of interest. In fact, one can say that $\langle K\rangle$ is defined by $\Gamma_{X P}$.

Another model that recasts preferential binding coefficient data in terms of a single model parameter is the local-bulk domain model developed by Courtenay et al [9]. The parameter in this model is the partition coefficient $K_{P}$, relating the 
number of water molecules and cosolvent molecules in the local and bulk domains via:

$$
K_{P}=\frac{n_{X}^{I I} / n_{W}^{I I}}{n_{X}^{I} / n_{W}^{I}}
$$

Similar to the site exchange model, the convention used in this model is that the local domain consists of a monolayer of water and enough cosolvent to obtain the experimentally observed $\Gamma_{X P}$. Note that because the absolute occupancy of water and cosolvent in the local domain cannot be easily determined by experiment, the local-bulk domain model effectively defines $n_{W}^{I I}$. Like $\langle K\rangle$, values of $K_{P}$ can be used to predict $\Gamma_{X P}$ at other cosolvent concentrations or for other proteins in the same cosolvent, but predictions cannot be made in the absence of $\Gamma_{X P}$ or free energy data on the same cosolvent system.

Lastly, transfer free energy models, pioneered by Bolen's group [15], take a different approach. These models conceptually divide whole proteins into groups [16] such as the amino acid side chains and the protein backbone and model the transfer free energy of the whole protein as a sum of the transfer free energy of the groups it comprises, via:

$$
\Delta \mu_{P}^{t r}=\sum_{i} \alpha_{i} \Delta g_{i}^{t r}
$$

where $\Delta g_{i}^{t r}$ is the transfer free energy of the model group and $\alpha_{i}$ is the solvent accessible area of the group in the whole protein, normalized to the solvent accessible area of the model compound. The overall $\Delta \mu_{P}^{t r}$ can then be predicted for any system of known structure. In the context of the previously described models, the transfer free energy model can be thought of as a linearized binding model where each surface group or amino acid in the protein represents a different type of independent binding site, and the binding constants for those sites are determined by experiments on model compounds, such as free amino acids or cyclic diamino acid compounds. Predictions made by transfer free energy models have met with mixed success. A linear group contribution model (equation 13) may be too simple to capture all of the important contributions to $\Delta \mu_{P}^{t r}[17]$.

While the above models have helped in the understanding of the phenomenon of preferential binding, they generally incorporate strong assumptions, and they necessitate the use of experimental data on highly analogous systems in order to determine model parameters and make predictions. Thus, their uses as predictive tools and as tools to gain insight into specific systems are limited.

In this work, we developed a predictive, molecular-level approach for the study of preferential binding based on allatom, statistical mechanical models that use no adjustable parameters. To date, statistical mechanical models of preferential binding have only been developed for interactions of ions with charged cylinders [18], [19] and for interactions of twodimensional, "hard circles" with a linear interface [20], both far too simple to be generally applied to protein-cosolvent systems. Other explicit mixed solvent simulations of proteins and amino acids have been performed [21], [22], [23], [24], [25], but these studies did not compute thermodynamic quantities

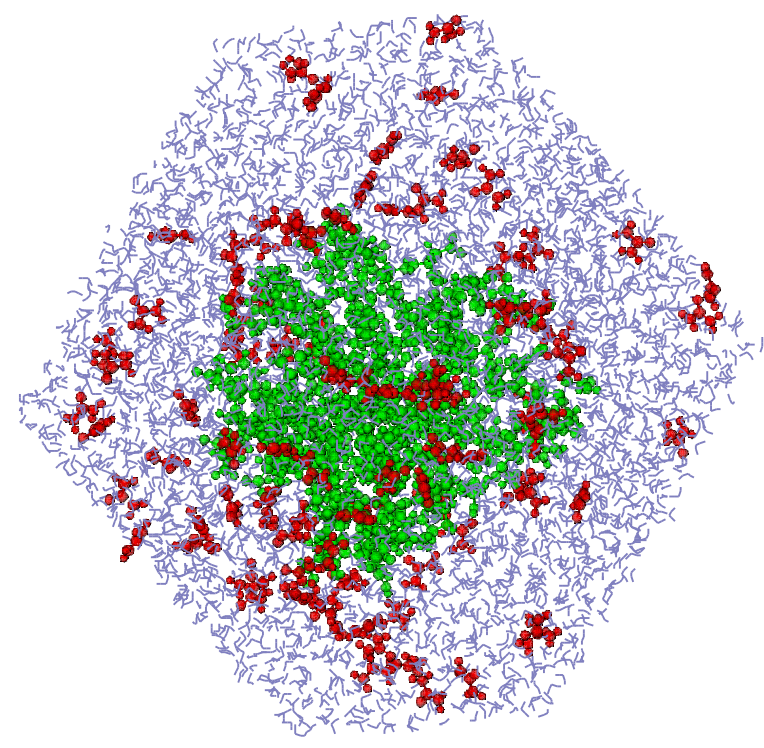

Fig. 2. A simulation cell containing RNase T1 (center, green spheres) solvated by water (thin blue lines) and urea (red spheres). Figure generated with VMD [28].

related to preferential binding. In our approach, we define the number of "bound" molecules in a thermodynamically consistent way and do not a priori incorporate any information about "binding sites." The use of our approach for the computation of preferential binding coefficients was validated in two systems by comparison with experimental data from the literature. Additionally, the molecular-level detail of the approach provides new insights into the following issues:

1) The changes in solvent and cosolvent concentration as a function of distance from the protein surface.

2) A precise definition of the "local domain" (Figure 1).

3) The differences in preferential binding or apparent binding equilibrium constant at different locations on the protein-solvent interface.

The success of this method in modeling preferential binding indicates that it captures the important underlying physics of protein-cosolvent-water systems and that the difficulty in quantitative prediction to date can be surmounted by explicitly incorporating the complex protein-solvent and solvent-solvent interactions.

\section{A. A New, Molecular-Level Approach to Computing Preferen- tial Binding}

Our approach uses explicit atomic interaction potentials (force fields), such as Lennard-Jones, Coulombic, spring, and torsion interactions, with pre-fit coefficients [26], [27]. Thermodynamic properties, such as preferential binding coefficients, are computed by averaging in the time domain via molecular dynamics (MD). A snapshot from a dynamic simulation of RNase T1 in a urea solution is shown in Figure 2. The results of such simulations contain all of the information needed to extract thermodynamic properties such as $\Gamma_{X P}$.

Molecular dynamics uses Newton's second law of motion, that acceleration is the quotient of force and mass, to compute the positions of each atom in the system as a function of time. 
To do this, an energy model, sometimes called a "force field," that can be used to compute the net force on any atom in any configuration is employed.

During the MD run, the positions of each atom are recorded at fixed intervals in time. These "snapshots" form an ensemble of configurations which can then be used to compute thermodynamic properties, such as $\Gamma_{X P}$.

Importantly, this method of computing $\Gamma_{X P}$ does not introduce any adjustable parameters to model preferential binding or any other aspect of a system containing a protein and two solvent components. All of parameters required by the MD method for energy computations are determined independently of this particular modeling objective, and in fact have been shown to be generally applicable to biological systems [29]. Thus, the method developed here could be used to estimate $\Gamma_{X P}$ and $\Delta \mu_{P}^{t r}$ in systems where no experimental data is available. It therefore facilitates the study of preferential binding when direct experimental study is difficult, such as at transition state configurations or at marginally stable states of proteins. Furthermore, it yields detailed, local, molecularlevel insight into the system studied.

Another benefit of this approach is that when equation 7 holds (such as for urea and glycerol), the protein transfer free energy $\left(\Delta \mu_{P}^{t r}\right)$ can be calculated from a single $\Gamma_{X P}$ simulation. Traditional free energy calculation methods such as thermodynamic integration [30], [31] require 15-20 trajectories, which is computationally difficult for protein systems of this size.

\section{B. Minimum Simulation Time}

Sufficient sampling of position-space configurations in time is required for the accurate calculation of $\Gamma_{X P}$ via equation 3 . Assuming that the average protein solution structure is close to that of the initial (crystal) structure and that water molecules sample position space rapidly because of their high density, the most important time scale to be captured is that of the cosolvents sampling position space. One way to estimate this time is that it must be much larger than the average time between cosolvent-cosolvent contacts.

An estimate of the time between contacts can be obtained as:

$$
t_{\text {contact }} \approx \frac{1}{12 \mathcal{D}}\left(\frac{V_{\text {solv }}}{n_{X}}\right)^{\frac{2}{3}}
$$

where $\mathcal{D}$ is the cosolvent diffusivity, $V_{\text {solv }}$ is the solvent volume, and $n_{X}$ is the number of cosolvent molecules. For the simulations performed here, the solvent is mostly water, so equation 14 can be further simplified to yield:

$$
t_{\text {contact }} \approx \frac{1}{12 \mathcal{D}}\left(\frac{1}{N_{A} \rho_{W} m_{X}}\right)^{\frac{2}{3}}
$$

where $N_{A}$ is Avogadro's number and $\rho_{W}$ is the density of water in $\mathrm{kg} / \mathrm{m}^{3}$. For a $1 \mathrm{~m}$ cosolvent in water system with a cosolvent diffusivity of $2 \times 10^{-9} \mathrm{~m}^{2} / \mathrm{s}$ (a lower bound on the diffusivities of the cosolvents studied here), $t_{\text {contact }}$ is about 30 ps. Thus, nanosecond trajectories will be required for good sampling of cosolvent position space. Importantly, this time increases as the cosolvent concentration decreases, implying that there is a minimum concentration that can be studied with any given amount of computational resources.

\section{Methodology}

\section{A. Molecular Simulations}

Molecular dynamics was used to sample the phase space of proteins solvated by water and a cosolvent. Version 28 of the CHARMM [26] molecular dynamics package was used for all simulations. The CHARMM force-field was used for the protein, and the TIP3P model [32] was used for water. A force-field was constructed for glycerol using the standard CHARMM geometries and partial charges for the atoms in a -CHOH- unit [26], [27]. Urea was assumed to be planar with bond lengths equal to the CHARMM standards and partial charges recomputed as done previously [33] but using the CHARMM van der Waals mixing rules in the objective function.

The structures of RNase A (PDB code: 1fs3) and RNase T1 (PDB code: 1ygw) were obtained from the Protein Data Bank [34]. In total, three simulations were performed: RNase A in $1 \mathrm{~m}$ glycerol ( $\mathrm{pH} 3)$, RNase $\mathrm{T} 1$ in $1 \mathrm{~m}$ glycerol $(\mathrm{pH} 7)$, and RNase $\mathrm{T} 1$ in $1 \mathrm{~m}$ urea ( $\mathrm{pH} 7)$. Details of each simulation are shown in Table I. Each protein was solvated in a trucated octahedral box extending a minimum of $9 \AA$ from the protein. The $\mathrm{pH}$ of each simulation was fixed by setting the protonation states of each ionizable side chain to the dominant form expected for each amino acid at the $\mathrm{pH}$ of interest. Arginine, cysteine, lysine, and tyrosine were protonated in all of the simulations. Aspartate, glutamate, and histidine were assumed to have pKa values of 3.4, 4.1, and 6.6 [35], [36], respectively, and were therefore protonated in the simulation at $\mathrm{pH} 3$ and deprotonated at $\mathrm{pH}$ 7. Initial placement of water and cosolvent molecules were random. Protein counterions were placed using SOLVATE 1.0. The system was first energy minimized at $0 \mathrm{~K}$, next heated to $298.15 \mathrm{~K}$, and then equilibrated for $1 \mathrm{~ns}$ in the NTP ensemble at one atmosphere. For the computation of the properties of interest, two nanoseconds of dynamics were then run, during which statistics were computed from snapshots of the trajectory every picosecond.

\section{B. Calculation of Preferential Binding Coefficients}

The trajectories were then used to define the local and bulk regions and compute $\Gamma_{X P}$ in the following manner. For the purpose of computing $\Gamma_{X P}$ and other thermodynamic and structural parameters, each water and cosolvent molecule was treated as a point at its center of mass. The distance of each of these points to the protein's van der Waals surface was computed, and then $\rho_{W}(r)$ and $\rho_{X}(r)$, defined as the number densities of these points at a distance $r$ from the protein, were computed. In all cases, the $\rho(r)$ functions exhibited peaks and valleys characteristic of solvation shells in the range $0<r<6 \AA$. At distances in the range of $6-8 \AA$ and higher, such variations are no longer seen, and the local number density is defined as bulk number density, $\rho(\infty)$. Such a region far from the protein containing a spatially uniform concentration of water and cosolvent must be present in the 


\begin{tabular}{ccccccc} 
Cosolvent & Protein & $\mathrm{T}\left({ }^{\mathrm{o}} \mathrm{C}\right)$ & $\mathrm{pH}$ & $n_{X}$ & $n_{W}$ & $\langle l\rangle(\AA)$ \\
\hline Urea & RNase T1 & 25 & 7 & 90 & 4274 & 57.48 \\
Glycerol & RNase T1 & 25 & 7 & 87 & 4582 & 59.24 \\
Glycerol & RNase A & 25 & 3 & 90 & 5480 & 62.86
\end{tabular}

TABLE I

DETAILS OF FOUR MOLECULAR DYNAMICS (MD) SIMULATIONS PERFORMED. $n_{X}$ IS THE NUMBER OF COSOLVENT MOLECULES; $n_{W}$ IS THE NUMBER OF WATER MOLECULES; AND < $l>$ IS THE AVERAGE DIMENSION OF THE PRIMARY UNIT CELL (WHICH VARIES DURING THE RUN AT CONSTANT PRESSURE).

simulation cell in order to define the local and bulk regions and calculate $\Gamma_{X P}$.

The position of the boundary between the local and bulk domains, a distance of $r_{*}$ away from the surface of the protein, was then determined by choosing the minimum distance at which no significant difference between $\rho\left(r_{*}\right)$ and $\rho(\infty)$ was apparent for either water or cosolvent. All solvent molecules whose centers of mass fell inside a distance of $r_{*}$ from the protein's van der Waals surface were defined as belonging to the local domain (II), and all other solvent molecules were defined as belonging to the bulk domain (I). With these definitions of the domains, the instantaneous preferential binding coefficient, $\Gamma_{X P}(t)$, was computed as

$$
\Gamma_{X P}(t) \equiv n_{X}^{I I}-n_{X}^{I}\left(\frac{n_{W}^{I I}}{n_{W}^{I}}\right)
$$

for each time point in each trajectory. The preferential binding coefficient, $\Gamma_{X P}$, was then computed for each trajectory as the time average of these instantaneous values:

$$
\Gamma_{X P}=\frac{1}{t} \int_{0}^{t} \Gamma_{X P}\left(t^{\prime}\right) d t^{\prime}
$$

The radial distribution functions $g_{X}(r)$ and $g_{W}(r)$ are defined as:

$$
g_{i}(r) \equiv \rho_{i}(r) / \rho_{i}(\infty)
$$

where $i$ represents water $(W)$ or a cosolvent $(X)$ species. These functions provide another route to compute $\Gamma_{X P}$ :

$$
\begin{aligned}
\Gamma_{X P}= & \left\langle n_{X}^{I I}\right\rangle-\left\langle\left(\frac{n_{X}^{I}}{n_{W}^{I}}\right) n_{W}^{I I}\right\rangle \\
= & \rho_{X}(\infty) \int g_{X} d V- \\
& \left(\frac{\rho_{X}(\infty)}{\rho_{W}(\infty)}\right) \rho_{W}(\infty) \int g_{W} d V \\
= & \rho_{X}(\infty) \int\left(g_{X}-g_{W}\right) d V
\end{aligned}
$$

where each integral is over the local domain or the entire system (since $g_{X}-g_{W}=0$ in the bulk domain).

The boundary between domains I and II must be placed far enough from the protein to ensure that it is in the bulk, yet at the smallest such distance so that statistical fluctuations in the number of molecules in the domains can be minimized. We can use the values of $g_{X}(r)$ and $g_{W}(r)$ to determine the optimal boundary. Defining $\Gamma_{X P}^{*}$ as the apparent preferential binding coefficient resulting from defining the local domain as those molecules whose centers of mass lie inside a distance $r_{*}$ from the protein:

$$
\Gamma_{X P}^{*}\left(r_{*}\right)=\rho_{X}(\infty) \int_{0}^{r_{*}}\left(g_{X}-g_{W}\right) \frac{d V}{d r} d r
$$

The error in $\Gamma_{X P}, E_{\Gamma}$, introduced by selecting a particular value of $r_{*}$ is then

$$
\begin{aligned}
E_{\Gamma} & =\Gamma_{X P}^{*}\left(r_{*}\right)-\Gamma_{X P} \\
& =-\rho_{X}(\infty) \int_{r_{*}}^{\infty}\left(g_{X}-g_{W}\right) \frac{d V}{d r} d r
\end{aligned}
$$

When $r_{*}$ is selected properly, the surface defined by $r=r_{*}$ is entirely in the bulk solution, $g_{X}\left(r_{*}\right)=g_{W}\left(r_{*}\right)=1$, and $E_{\Gamma}=0$. Thus, selecting $r^{*}$ as the minimum distance for which all $r \geq r^{*}$ satisfy $g_{X}(r)=g_{W}(r)=1$ (within the error of the simulation) is optimal.

\section{Estimation of Statistical Error}

The statistical error arising from computing averaged properties from a finite trajectory was estimated in the following fashion:

1) The dynamic trajectory of interest was divided into $n$ pieces.

2) The mean of the property of interest was computed in each piece. These means were designated $\bar{x}_{i}$ where $i=$ 1..n.

3) The standard deviation of the $\bar{x}_{i}$ values was computed.

4) This standard deviation was divided by $\sqrt{n}$ and the quotient was designated $\sigma_{m}$, an estimate of the error in the mean determined by time averaging the full trajectory.

The number of pieces $n$ into which the trajectory is divided must be small enough to ensure that the means of each piece (the $\bar{x}_{i}$ ) are statistically independent. An autocorrelation analysis (not shown) of several trajectories of $\Gamma_{X P}(t)$ data and the underlying molecular counts $\left(n_{i}^{I}\right.$ and $\left.n_{i}^{I I}\right)$ indicates that a window of about $0.2 \mathrm{~ns}$ is sufficiently large for this to be true. Therefore, for a $2 \mathrm{~ns}$ dynamics trajectory, a value of $n=2 / 0.2=10$ was used.

For long trajectories, the statistical error $\sigma_{m}$ is roughly proportional to the inverse square root of the trajectory length. This property can be used to estimate the trajectory length required to achieve a given level of statistical accuracy after a small trajectory has been generated and analyzed.

\section{RESUlTS AND Discussion}

\section{A. Radial Distribution Functions of Water and Cosolvents}

The radial distribution functions of water, urea, and glycerol were computed for all three simulations as described in Methodology and are shown in Figure 3.

At very short distances, $r<0.6 \AA$ for water and $r<1.0 \AA$ for glycerol and urea, regions of total solvent and cosolvent 

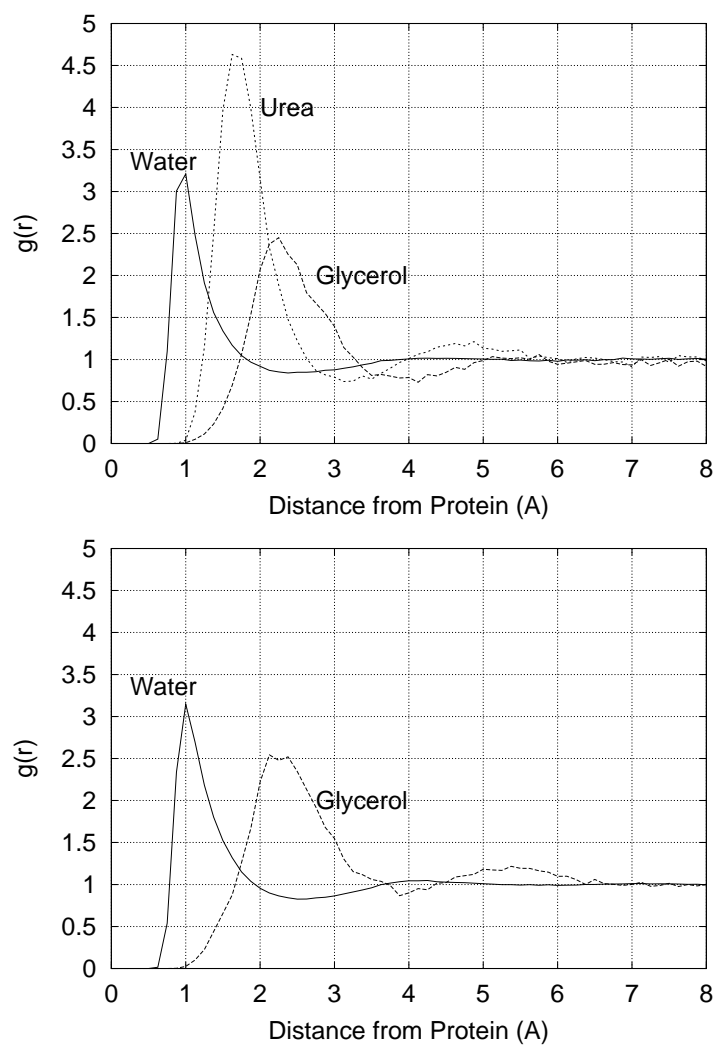

Fig. 3. Radial distribution functions of water, urea, and glycerol are shown for simulations of RNase T1 in glycerol and urea solutions (left) and RNase A in a glycerol solution (right). In the left-hand figure, the difference between the two $g_{W}(r)$ functions is not visible at this scale.

exclusion due to very strong van der Waals repulsion can be seen. The size of these "totally excluded" regions is much smaller than one would expect based on the apparent van der Waals radii of the solvent and cosolvent molecules alone (for example, $r \approx 1.5 \AA$ for water and $2.2 \AA$ for urea [37]), indicating that electrostatic attractive forces play an important role in solvation even at these distances. After the regions of total exclusion, strong first coordination shells of these three molecules can be clearly seen. The peaks of the first coordination shells become more distant from the protein as the size of the molecules they correspond to increases. Significantly smaller second coordination shell peaks are also visible for urea solvating RNase $\mathrm{T} 1$ and glycerol solvating RNase A. At distances greater than $6-7 \AA$ from the protein, solvation shells cannot be discerned, and the number densities of water, urea, and glycerol reach their bulk values.

In the simulations of RNase $\mathrm{T} 1$ in glycerol and urea solutions, the radial distribution functions for glycerol and urea are quite different. The maximum value of $g_{X}(r)$ for urea is over 4.5, while that for glycerol is about 2.5 . The difference in these maximum values, while significant, is not sufficient to say that the number of urea molecules coordinated to the protein $\left(n_{X}^{I I}\right)$ is higher than the number of glycerol molecules coordinated; this can only be done by integrating each $g_{X}(r)$ function appropriately via equation 19 .

The radial distribution functions for both water and glycerol are similar in the simulations of RNase A and RNase T1 in

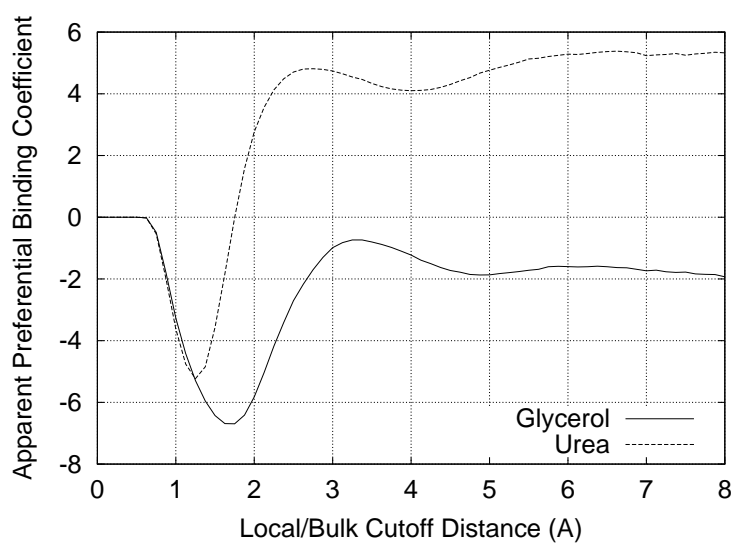

Fig. 4. Apparent preferential binding coefficient as a function of the cutoff distance between the local and bulk domains for simulations of RNase T1 in glycerol and urea solution.

glycerol solution, despite the fact that the proteins and the $\mathrm{pHs}$ of the solutions are different. Given that the proteins are of similar size, this observation is consistent with the fact that the values of $\Gamma_{X P}$ for the two solutions are close.

\section{B. Preferential Binding Coefficients}

The radial distribution functions in Figure 3 suggest that $r_{*}$ in the range of $6-8 \AA$ is an appropriate choice of boundary between the local and bulk domains. The error in $\Gamma_{X P}$ introduced by a particular choice of the boundary distance, $r_{*}$, can be estimated by plotting the apparent preferential binding coefficient $\left(\Gamma_{X P}^{*}\right)$ versus $r_{*}$ (Figure 4$)$. $\Gamma_{X P}^{*}$ depends very strongly on $r_{*}$ in the first solvation shell $(r=0-4 \AA)$ and weakly on $r_{*}$ in the second solvation shell $(r=4-6 \AA)$. In the range $r=6-8 \AA$, the dependence of $\Gamma_{X P}^{*}$ on $r_{*}$ is small $\left( \pm 0.5\right.$ ), and is less than the statistical error in $\Gamma_{X P}$ (shown in Table II, explained below). Therefore, a cutoff distance of $6 \AA$, or about two solvation shells, is sufficiently large to minimize systematic error in $\Gamma_{X P}$ caused by the choice of $r_{*}$. If only a single solvation shell were considered $\left(r_{*} \sim 3.5-4 \AA\right)$, a systematic error in $\Gamma_{X P}$ of approximately $0.5-1$ molecules would be introduced as a result of neglect of the second solvation shell.

The preferential binding coefficient, $\Gamma_{X P}$, was computed via equation 3 using $r_{*}=6 \AA$ as the boundary between the local and bulk domains. A confidence interval for this ensemble average was computed as described in Methodology. The binding coefficients and their statistical uncertainties are shown in Table II. Experimental values from the literature were available for two out of three of these protein-cosolvent systems, and our computed values of $\Gamma_{X P}$ agree quite favorably with these. The fact that this occurs for both positive and negative values of $\Gamma_{X P}$ without the use of any adjustable parameters is very encouraging. For a cosolvent that obeys equation 7 , the confidence intervals of \pm 1.0 in $\Gamma_{X P}$ represents a confidence limit in the transfer free energy of about $0.6 \mathrm{kcal} / \mathrm{mol}$, which is a typical value for free energies calculated via this type of molecular simulation. Achievement of this level of accuracy despite the fact that structural fluctuations in the native state ensemble of proteins have been observed on much longer time 


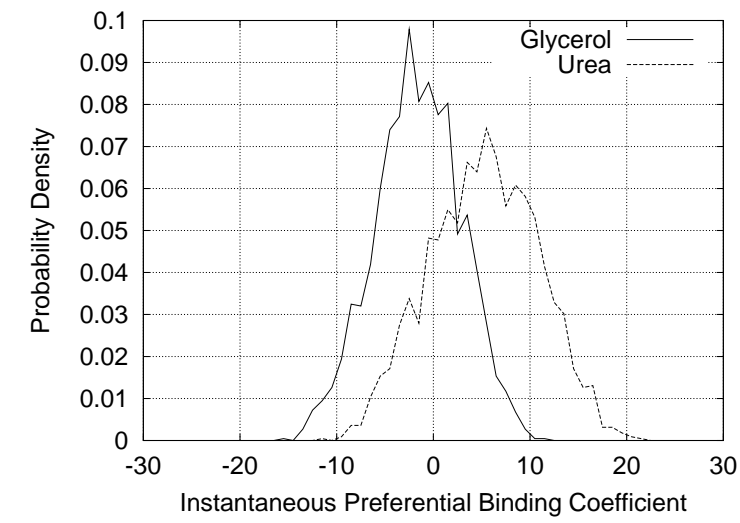

Fig. 5. $\Gamma_{X P}(t)$ probability density function. A wide range of values of $\Gamma_{X P}(t)$ are sampled as water and cosolvent molecules diffuse between the local and bulk domains.

scales [38] than the time scale of the simulations performed here suggests that solvent dynamics are more important than protein structural dynamics in determining $\Gamma_{X P}$.

$\Gamma_{X P}(t)$ probability density functions for the simulations of RNase T1 in urea and glycerol solution are shown in Figure 5. The range of instantaneous values of the preferential binding coefficient, $\Gamma_{X P}(t)$, is quite large relative to the absolute values of $\Gamma_{X P} \cdot \Gamma_{X P}(t)$ values in excess of $\Gamma_{X P} \pm 15$ are observed. The breadths of these distributions are related to the size of the interface between the local and bulk domains and indicate the importance of sampling a large number of solvent configurations to obtain the macroscopic, averaged $\Gamma_{X P}$ (equation 17).

\section{The Relation between Solvent Accessible Area and the Number of Molecules in the Local Domain}

The solvent accessible areas of whole proteins (SAA) and constituent groups $\left(\mathrm{SAA}_{i}\right)$ in crystal structures have been used extensively in analyzing proteins. SAA and $\mathrm{SAA}_{i}$ are essentially simple ways of measuring water coordination numbers. In models developed to date, SAA or $\mathrm{SAA}_{i}$ has been used to estimate $n_{W}^{I I}$ or $n_{W, i}^{I I}$ by assuming that the local domain is a monolayer of water and each water molecule occupies approximately $10 \AA^{2}$ of the solvent accessible area. Since we have introduced a new notion of the local domain, it is worthwhile to see what relationships exist between $\mathrm{SAA}_{i}$ and the coordination numbers $n_{W, i}^{I I}$ and $n_{X, i}^{I I}$ that utilize this definition.

A scatter plot of the solvent accessible area of a set of constituent groups (amino acid side chains and the protein backbone) versus the number of water molecules in the local domain for three different simulations is shown in Figure 6. Solvent accessible area was calculated analytically in CHARMM (based on Richmond's method [40]) using a $1.4 \AA$ probe. There is a strong, linear correlation of these variables with slope $4.2 \AA^{2} /$ molecule and correlation coefficient 0.96 . Similarly strong correlations are seen for $\mathrm{SAA}_{i}$ with $n_{X, i}^{I I}$ in individual simulations. A summary of proportionality constants and correlation coefficients for these relationships is shown in Table III. If the time average $\mathrm{SAA}_{i}$ from each

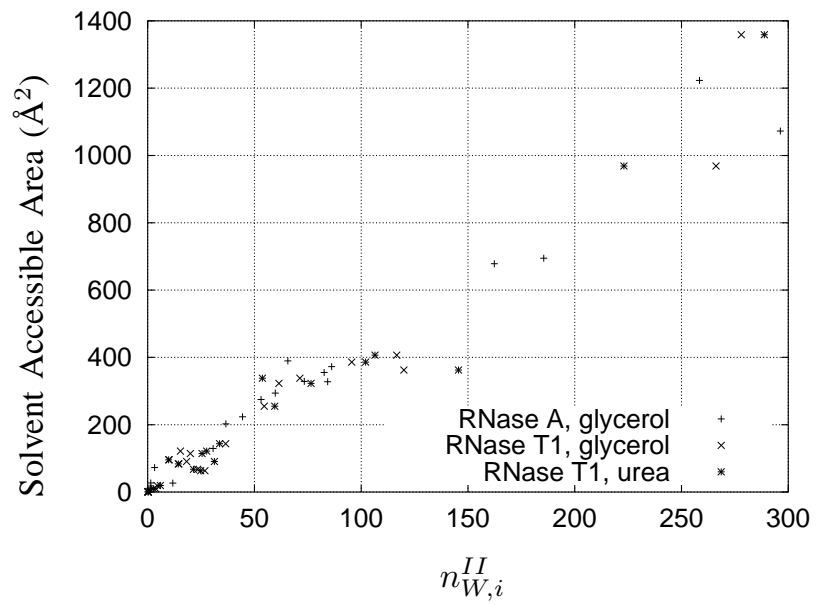

Fig. 6. Correlation of solvent accessible area and the number of water molecules in the local domain of constituent groups. Each point represents a constituent group of either a type of amino acid side chain or the protein backbone in one of the three simulations shown in Table II. The solvent accessible area of a constituent group and the number of water molecules in the local domain of the solvent near the group $\left(n_{W, i}^{I I}\right)$ are highly correlated.

dynamics simulation is used instead of the crystal structure $\mathrm{SAA}_{i}$ values, the correlation coefficients increase slightly. Because the time average solvent accessible areas are higher than those in the crystal structure, the proportionality constants shown in Table III also increase.

\section{CONCLUSIONS}

A quantitative method based on molecular dynamics simulations using all atom potential models has been developed and validated for calculating preferential binding coefficients. Our method is not a derivative of thermodynamic integration or thermodynamic perturbation methods and requires only a single trajectory to compute the transfer free energy of a protein into a weak-binding cosolvent system. Our results match experimental data well for glycerol and urea solutions, covering a range of positive and negative binding behavior. This work also augments experimentally-observable, macroscopic thermodynamics with the mechanistic insight provided by a molecular-level, statistical mechanical model.

Variations in the radial distribution functions with distance for each cosolvent are evident up to about $6 \AA$, or two solvation shells of water, away from the protein. Glycerol is not totally excluded from close contact with the protein, but glycerol is less likely than urea to be found in such a position. The radial distribution functions of water and cosolvents are sufficient to calculate preferential binding coefficients by integrating over a suitable solvent volume.

\section{REFERENCES}

[1] S. N. Timasheff, "Control of protein stability and reactions by weakly interacting cosolvents: The simplicity of the complicated," Adv. Protein Chem., vol. 51, pp. 355-431, 1998.

[2] J. C. Lee and S. N. Timasheff, "The stabilization of proteins by sucrose," J. Biol. Chem., vol. 256, no. 14, pp. 7193-7201, 1981.

[3] J. G. Kirkwood and R. J. Goldberg, "Light scattering arising from composition fluctuations in multi-component systems," J. Chem. Phys., vol. 18 , no. 1 , pp. $54-57,1950$. 


\begin{tabular}{l|c|c|c} 
& & Simulation & Experiment \\
System & $m_{\text {bulk }}$ & $\Gamma_{X P}$ & $\Gamma_{X P}$ \\
\hline Urea / RNase T1 & $1.10 \mathrm{~m}$ & $5.2 \pm 1.0$ & $6.4[39]$ \\
Glycerol / RNase T1 & $1.07 \mathrm{~m}$ & $-1.6 \pm 0.8$ & \\
Glycerol / RNase A & $0.91 \mathrm{~m}$ & $-0.9 \pm 1.0$ & $-1.7 \pm 0.8[6]$
\end{tabular}

TABLE II

PREFERENTIAL BINDING COEFFICIENTS COMPUTED FROM MD SIMULATIONS AND COMPARED WITH AVAILABLE EXPERIMENTAL DATA AT SIMILAR COSOLVENT CONCENTRATIONS. A WIDE RANGE OF BEHAVIOR (POSITIVE AND NEGATIVE PREFERENTIAL BINDING COEFFICIENTS) CAN BE MODELED WITHOUT THE USE OF ADJUSTABLE PARAMETERS. THE CONFIDENCE INTERVALS ON $\Gamma_{X} P(\mathrm{MD})$ ARE AN ESTIMATE OF THE STATISTICAL ERROR RESULTING FROM THE USE OF A FINITE TRAJECTORY. FOR EASIER COMPARISON, THE EXPERIMENTAL VALUES OF $\Gamma_{X P}$ REPORTED ABOVE WERE INTERPOLATED TO $m_{b u l k}$ FROM DATA SETS SPANNING THE MOLALITY OF INTEREST.

\begin{tabular}{c|c|c|c} 
Species $(i)$ & Protein & $\begin{array}{c}\text { Avg Protein SAA } / n_{i}^{I I} \\
\left(\AA^{2} / \text { molecule }\right)\end{array}$ & $r^{2}$ \\
\hline Water & RNase A/T1 & 4.2 & 0.96 \\
$0.91 \mathrm{~m}$ Glycerol & RNase A & 290 & 0.96 \\
$1.07 \mathrm{~m}$ Glycerol & RNase T1 & 230 & 0.93 \\
$1.10 \mathrm{~m}$ Urea & RNase T1 & 170 & 0.98
\end{tabular}

TABLE III

RELATIONSHIPS BETWEEN SOLVENT ACCESSIBLE AREA IN EACH PROTEIN CRYSTAL STRUCTURE AND NUMBER OF SOLVENT MOLECULES IN THE LOCAL DOMAIN FOR DIFFERENT PROTEIN-COSOLVENT SYSTEMS. $r^{2}$ SYMBOLIZES THE CORRELATION COEFFICIENT.

[4] J. A. Schellman, "Solvent denaturation," Biopolymers, vol. 17, pp. 1305$1322,1978$.

[5] J. C. Lee and S. N. Timasheff, "Partial specific volumes and interactions with solvent components of proteins in guanidine hydrochloride," Biochemistry, vol. 13, pp. 257-265, 1974.

[6] K. Gekko and S. N. Timasheff, "Mechanism of protein stabilization by glycerol: Preferential hydration in glycerol-water mixtures," Biochemistry, vol. 20, pp. 4667-4676, 1981.

[7] _ " "Thermodynamic and kinetic examination of protein stabilization by glycerol," Biochemistry, vol. 20, pp. 4677-4686, 1981.

[8] N. Poklar, N. Petrovcic, M. Oblak, and G. Vesnaver, "Thermodynamic stability of ribonuclease a in alkylurea solutions and preferential solvation changes accompanying its thermal denaturation: A calorimetric and spectroscopic study," Protein Sci., vol. 8, pp. 832-840, 1999.

[9] E. S. Courtenay, M. W. Capp, C. F. Anderson, and M. T. Record Jr., "Vapor pressure osmometry studies of osmolyte-protein interactions: Implications for the action of osmoprotectants in vivo and for the interpretation of osmotic stress experiments in vitro," Biochemistry, vol. 39, pp. 4455-4471, 2000.

[10] E. F. Casassa and H. Eisenberg, "Thermodynamic analysis of multicomponent solutions," Adv. Protein Chem., vol. 19, pp. 287-395, 1964.

[11] R. F. Greene Jr. and C. N. Pace, "Urea and guanidine hydrochloride denaturation of ribonuclease, lysozyme, alpha-chymotrypsin, and betalactoglobulin," J. Biol. Chem., vol. 249, no. 17, pp. 5388-5393, 1974.

[12] M. T. Record Jr., W. Zhang, and C. F. Anderson, "Analysis of effects of salts and uncharged solutes on protein and nucleic acid equilibria and processes: A practical guide to recognizing and interpreting polyelectrolyte effects, hofmeister effects, and osmotic effects of salts," Adv. Protein Chem., vol. 51, pp. 281-353, 1998.

[13] J. Wyman and S. J. Gill, Binding and Linkage: Functional Chemistry of Biological Macromolecules. University Science Books, 1990.

[14] J. A. Schellman, "Fifty years of solvent denaturation," Biophys. Chem., vol. 96, pp. 91-101, 2002.

[15] Y. F. Liu and D. W. Bolen, "The peptide backbone plays a dominant role in protein stabilization by naturally-occurring osmolytes," Biochemistry, vol. 34 , no. 39, pp. 12884-12 891, 1995.

[16] C. Tanford, "Isothermal unfolding of globular proteins in aqueous urea solutions," J. Am. Chem. Soc., vol. 86, pp. 2050-2059, 1964.

[17] D. W. Bolen, "Protein stabilizaiton by naturally occurring osmolytes," in Protein Structure, Stability, and Folding. Humana Press, 2001.

[18] C. F. Anderson and M. T. Record Jr., "Salt dependence of oligoionpolyion binding: A thermodynamic description based on preferential interaction coefficients," J. Phys. Chem., vol. 97, no. 27, pp. 7116-7126, 1993.

[19] P. Mills, C. F. Anderson, and M. T. Record Jr., "Grand canonical monte carlo calculations of thermodynamic coefficients for a primitive model of dna-salt solutions," J. Phys. Chem., vol. 90, no. 24, pp. 6541-6548, 1986.

[20] K. E. S. Tang and V. A. Bloomfield, "Assessing accumulated solvent near a macromolecular solute by preferential interaction coefficients," Biophys. J., vol. 82, pp. 2876-2991, 2002.

[21] Q. Zou, B. J. Bennion, V. Daggett, and K. P. Murphy, "The molecular mechanism of stabilization of proteins by tmao and its ability to counteract the effecfts of urea," J. Am. Chem. Soc., vol. 124, no. 7, pp. 1192-1202, 2002.

[22] B. J. Bennion and V. Daggett, "The molecular basis for the chemical denaturation of proteins by urea," PNAS, vol. 100, no. 9, pp. 5142-5147, 2003.

[23] J. Tirado-Rives, M. Orozco, and W. L. Jorgensen, "Molecular dynamics simulations of the unfolding of barnase in water and $8 \mathrm{~m}$ aqueous urea," Biochemistry, vol. 36, pp. 7313-7329, 1997.

[24] D. O. V. Alonso and V. Daggett, "Molecular dynamics simluations of protein unfolding and limited refolding: Characterization of partially unfolded states of ubiquitin in 60vol. 247, pp. 501-520, 1995.

[25] A. Caflisch and M. Karplus, "Structural details of urea binding to barnase: A molecular dynamics analysis," Struct. Fold. Des., vol. 7, pp. 477-488, 1999.

[26] B. R. Brooks, R. E. Bruccoleri, B. D. Olafson, D. J. States, W. Swaminathan, and M. Karplus, "Charmm: A program for macromolecular energy, minimization, and dynamics calculations," J. Comp. Chem., vol. 4, no. 2, pp. 187-217, 1983.

[27] S. N. Ha, A. Giammona, M. Field, and J. W. Brady, "A revised potentialenergy surface for molecular mechanics studies of carbohydrates," Carbohydrate Res., vol. 180, pp. 207-221, 1988.

[28] W. Humphrey, A. Dalke, and K. Schulten, "Vmd - visual molecular dynamics," J. Molec. Graphics, vol. 14, no. 1, pp. 33-38, 1996.

[29] M. Karplus and J. A. McCammon, "Molecular dynamics simulations of biomolecules," Nature. Struct. Biol., vol. 9, pp. 646-652, 2002.

[30] P. A. Bash, U. C. Singh, R. Langridge, and P. A. Kollman, "Free energy calculations by computer simluation," Science, vol. 236, pp. 564-569, 87.

[31] P. Kollman, "Free energy calculations: Applications to chemical and biochemical phenomena," Chem. Rev., vol. 93, pp. 2395-2417, 1993.

[32] W. L. Jorgensen, J. Chandrasekhar, J. D. Madura, R. W. Impey, and M. L. Klein, "Comparison of simple potential functions for simulating liquid water," J. Chem. Phys., vol. 79, no. 2, pp. 926-935, 1983.

[33] E. M. Duffy, D. L. Severance, and W. L. Jorgensen, "Urea: Potential functions, $\log$ p, and free energy of hydration," Israel J. Chem., vol. 33, no. 3, pp. 323-330, 1993.

[34] H. M. Berman, J. Westbrook, Z. Feng, G. Gilliand, T. N. Bhat, H. Weissig, I. N. Shindyalov, and P. E. Bourne, "The protein data bank," Nucleic Acids Res., vol. 28, pp. 235-242, 2000. 
[35] W. R. Forsyth, J. M. Antosiewicz, and A. D. Robertson, "Empirical relationships between protein structure and carboxyl pka values in proteins," Proteins, vol. 48, pp. 388-403, 2002.

[36] S. P. Edgecomb and K. P. Murphy, "Variability in the pka of histidine side-chains correlates with burial within proteins," Proteins, vol. 49, pp. $1-6,2002$.

[37] J. A. Schellman, "Protein stability in mixed solvents: A balance of contact interaction and excluded volume," Biophys. J., vol. 85, no. 1, pp. 108-125, 2003.

[38] Y. Duan and P. A. Kollman, "Pathways to a protein folding intermediate observed in a 1-microsecond simulation in aqueous solution," Science, vol. 282, pp. 740-744, 1998.

[39] T. Y. Lin and S. N. Timasheff, "Why do some organisms use a ureamethylamine mixture as osmolyte- thermodynamic compensation of urea and trimethylamine n-oxide interactions with protein," Biochemistry, vol. 33, no. 42, pp. 12695-12 701, 94.

[40] T. J. Richmond, "Solvent accessible surface-area and excluded volume in proteins- analytical equations for overlapping spheres and implications for the hydrophobic effect," J. Mol. Biol., vol. 178, no. 1, pp. 63-89, 1984. 